CIC. Cuadernos de Información y Comunicación ISSN: 1135-7791

http://dx.doi.org/10.5209/CIYC.55970

\title{
Tecnología e identidad: el caso de los Inuit y Facebook
}

\author{
Alexander Castleton ${ }^{1}$
}

Enviado: 20 de abril 2017 / Aceptado: 25 de abril de 2017

Resumen. Este artículo discute la relación entre tecnología, identidad cultural, y cambio social en el ártico canadiense. Utilizando una estrategia etnográfica basada en entrevistas y observación de contenidos digitales, se explora cómo un grupo de estudiantes del centro terciario de estudios técnicos "Arctic College" localizado en la comunidad de Iqaluit, en la isla Baffin del ártico canadiense, usa la red social Facebook. Se halló que, además de un uso esperado de la red social asociada al "guión" que promueve la tecnología, los jóvenes Inuit usan Facebook para acceder a contenidos relacionados a su identidad en diversos grupos, discutir asuntos culturales, y recordar tradiciones. Este artículo sostiene que la identidad Inuit es un ejemplo de cómo las culturas indígenas tienen que ser entendidas como algo dinámico, en constante cambio, para las cuales las TIC son fundamentales. El ser Inuit incluye artefactos como computadoras, internet o Facebook, para la afirmación y práctica de su cultura.

Palabras clave: Identidad; TIC; Facebook; Inuit.

\section{[en] Technology and identity: the case of the Inuit and Facebook}

Abstract. This article discusses the relationship between technology, cultural identity, and social change in the Canadian Arctic. Using an ethnographic strategy based on interviews and digital content observation, I explore how a group of students from the Arctic College located in the community of Iqaluit located on Baffin Island in the Canadian Arctic, use the social networking site Facebook. I found that, in addition to an expected use of the social network associated with the script of the technology, Inuit youth use Facebook to access content related to their identity, discuss cultural issues, and recall traditions. This article argues that the Inuit identity is an example of how indigenous cultures have to be understood as something dynamic and in constant change for which ICTs are fundamental. Inuit identity includes artifacts such as computers, the Internet or Facebook for the affirmation and practice of their culture.

Keywords: Identity; ICT; Facebook; Inuit.

Sumario. 1. Introducción. 2. Metodología. 3. Los inuit, el ártico canadiense, y las tic. 4. Descifrando la identidad inuit. 5. Uso individual de facebook. 6. Uso de grupos de facebook. 7. Conclusión: tecnología e identidad. Bibliografía.

Cómo citar: Castleton, A. (2017). Tecnología e identidad: el caso de los Intuit y Facebook, en CIC. Cuadernos de Información y Comunicación 22, 107-125.

1 Doctorando en Sociología por Carleton University (Ottawa, Canadá).

Email: alexcastlef@gmail.com 


\section{Introducción}

Este artículo discute la relación entre tecnología, identidad y cambio social en el ártico canadiense. Se explora en el uso de tecnologías de la información y comunicación (TICs) y jóvenes Inuit. Se examina, específicamente, cómo jóvenes Inuit entre 18 a 28 años estudiantes del centro de estudios terciarios Arctic College en Iqaluit (comunidad situada en la isla Baffin, parte del territorio de Nunavut en el norte de Canadá), utilizan la red social Facebook en el contexto de su vida diaria y cómo su uso se relaciona con su identidad Inuit.

La forma en que poblaciones indígenas alrededor del mundo usan TICs ha sido discutida en varios volúmenes (por ejemplo, Dyson et al. 2015; Landzelius 2006; Dyson et al. 2006), en múltiples artículos académicos (por ejemplo, Molyneaux, et al, 2012; Carpenter, et al. 2013; McMahon, 2013; Srinivasan, 2006, 2012; Taylor, 2012, Young-Ing, 2003) y no académicos (por ejemplo, Taylor, 2011; Krebs, 2011). Estos autores y críticos básicamente han explorado la apropiación de TICs para establecer y negociar identidad en un mundo globalizado, y como herramientas que pueden ser utilizadas para la transmisión y conservación cultural ${ }^{2}$. Por otro lado, algunas voces críticas han remarcado que podría existir un lado no tan beneficioso del uso de TICs relacionado con la "exotizacion" de las poblaciones indígenas que terminarían por promover una suerte de colonización cultural online (Ginsburg, 1991; Krone, 2007, 2008; Santo, 2004, 2008; Nathan, 2000; Fair, 2000; Ess, 2005), y la pérdida de conocimientos y habilidades tradicionales (Aporta y Higgs, 2005).

En relación a los Inuit específicamente, algunos observadores han notado los beneficios que las TICs les otorgan considerando el contexto sociocultural y económico tan cambiante del ártico (Scobie y Wachowich, 2010; Rodgers y Scobie, 2015; Alexander, 2011; Alexander et al. 2009). El ártico es un ambiente donde los Inuit han logrado florecer durante miles de años. A pesar de un proceso de colonización terrible que involucró el intento de total asimilación a la cultura colonizadora, los Inuit han logrado salir de manera relativamente satisfactoria del proceso de colonización, por ejemplo, negociando en los años 90 con el gobierno de Canadá el tener su territorio autónomo, Nunavut $^{3}$ (Dahl et al. 2000).

Desde el primer contacto con los europeos, los Inuit han incorporado tecnologías foráneas a sus prácticas culturales tradicionales, siendo el más claro ejemplo el rifle, utilizado para cazar (Damas, 2000; Tester, 2010). De la mano de estos cambios técnicos, es imprescindible tener en cuenta que como Soukoup (2006: 240) ha escrito, los Inuit han sufrido una muy rápida transición de "la prehistoria a la era atómica en una generación". En este contexto es importante considerar cómo las nuevas generaciones negocian su propia identidad como Inuit con las nuevas TIC que ya son practicamente ubicuas en el ártico canadiense. Evidencia de esto es lo que el anciano John Amagoalik, quien fue uno de los artífices del territorio Inuit de Nunavut, notaba de modo un tanto pesimista para el periódico Nunatsiaq news (2014) de Nunavut:

Un ejemplo de esto es un mapa que interactivo online que documenta la compleja red de caminos existentes a través del Ártico que los Inuit utilizan desde tiempos inmemoriales. (Rogers, 2013).

3 El proceso de colonización de los pueblos indígenas de Canadá no se puede minimizar. Uno de los hechos más traumáticos fue el sistema de escuelas residenciales donde los niños eran llevados, arrancados de sus comunidades, obligados a aprender las costumbres occidentales por medio de maltratos y abusos. Un gran número de niños murió producto de los abusos o por enfermedades como la tuberculosis. (Rogers at al. 2012) 
"Hoy tenemos una nueva adicción 4 invadiendo nuestra sociedad. Se llama la era de la tecnología. El world wide web, Internet, computadoras, Smart phones, tablets,iPods, iPads, Facebook, Twitter, Google, sitios web, podcasts, juegos electrónicos online, jihad, religión, odio, pornografía, romance, etc., etc., etc."

En este marco, en este artículo examino el uso de una herramienta que las TIC traen: la red social Facebook.

\section{Metodología}

Esta investigación es el producto de una estrategia etnográfica basada en observaciones, entrevistas, y análisis de contenido digital durante el otoño del 2014 en Iqaluit, Nunavut. Pasé cinco semanas viviendo en la residencia del Arctic College, un centro de enseñanza politécnica en la comunidad Iqaluit donde conviví e interactúe con jóvenes Inuit de diferentes comunidades del vasto territorio de Nunavut. Desarro1lé lo que Clifford Geertz (1998) describió como deep hanging-out, que condujo a entrevistas y conversaciones, que revelaron la importancia que Facebook tiene para los jóvenes Inuit participantes. Esto provocó que en el trabajo de campo gravitara a explorar dicha red social, por lo que las observaciones in situ y las entrevistas fueron acompañadas de observaciones digitales del uso de Facebook de los entrevistados. La importancia de esta estrategia se hace evidente si consideramos como sostuvieron Wilson et al. (2012) que los perfiles de Facebook son una especie de lienzo en blanco donde los usuarios tienen libertad de construir una imagen pública o semi-pública, y que por lo tanto otorga nuevos insumos para pensar la formación de identidad. Por lo tanto, el uso de Facebook es interesante en la medida que los jovenes Inuit desplegaban ahí su vida diaria e intereses, haciéndolo un lugar adecuado para estudiar la relación entre tecnología e identidad.

Mi proceso de integración a la vida diaria de la residencia fue casi inmediato, y generé instantáneo rapport con los estudiantes. Ayudó el hecho de que a pesar de ser blanco, no soy lo que se podría decir $100 \%$ canadiense, lo cual fue revelado por un acento al hablar inglés que no es de un canadiense típico. Este hecho fue una ventaja en el sentido de que hay cierto recelo por el proceso de colonización, y mi condición de inmigrante reciente al país provocó que los estudiantes estuvieran tan interesados por mi cultura como yo por la de ellos.

También fueron insumo metodológico herramientas de la teoría fundada (ver Charmaz, 2006), especialmente la comparación constante de los datos que surgían a medida que desarrollaba el trabajo de campo. Estos permitieron destacar dos usos fundamentales de Facebook: uno individual, más evidente dado el "guión" o "la invitación al uso"s que hace Facebook, y otro más complejo en torno a grupos formados dentro de la red social.

Amagoalik dice una "nueva" adicción por los índices altísimos de abuso de sustancias existente en Nunavut y entre los Inuit en general.

5 Por "invitación al uso" refiero al concepto de affordances descrito por el psicólogo J.J Gibson (1977), que refiere a las posibilidades de uso que una tecnología otorga. 


\section{Los Inuit, el ártico canadiense, y las TIC}

Dicks (1977) fue uno de los pioneros en discutir el impacto de la introducción de TIC en el ártico al reflexionar sobre el teléfono de dial. Este autor sugirió que "el desarrollo de sistemas de telecomunicación, mientras que presenta nuevas opciones facilitando el contacto personal, también ha traído problemas para la forma de vida tradicional de los Inuit" (p. 120). Lo que este autor sostuvo hace 40 años apunta a que la incorporación histórica de tecnología es de cierto modo el chivo expiatorio para los problemas que padecen los Inuit, quienes no podrían estar culturalmente a tono con cambios tecnológicos de esta magnitud. Dicks defiende su hipótesis de modo Durkheimiano por medio de cifras que demostraban el incremento en las tasas de crímenes a partir de los cambios tecnológicos. No obstante, al mismo tiempo reconoce las nuevas oportunidades que una tecnología de comunicación como el teléfono trae para la población ártica, incluyendo la creación de asociaciones, facilitando la comunicación con el gobierno federal, y entre grupos de interés político territoriales (Dicks, 1977: 129).

Posteriormente y de modo similar, Savard (1998) mostró un cariz crítico en cuanto a la incipiente introducción de internet en Nunavut. Por un lado, sugirió los beneficios de la tecnología: promocionar el contacto entre una población dispersa, la cohesión social, la coordinación gubernamental, y favorecer la economía. Pero también entiende que "[1] os Inuit de Nunavut no están protegidos de los impactos de uso de Internet. Por el contrario, corren el riesgo de ser las primeras víctimas de la meta-cultura cibernética. Los jóvenes cibernautas Inuit van a ver sus valores aborígenes confrontados con valores no-aborígenes, aquellos colonizando a estos" (Savard, 1998: 89). Y más adelante también afirma este autor:

...la herramienta usada en el ciberespacio, la computadora, es la prolongación de otra herramienta europea, la escritura (...) Por lo tanto, no están los Inuit solo obligados a apropiarse de una herramienta que es centralmente foránea a ellos, sino que también están confrontados con valores que son radicalmente diferentes de los suyos (...) Los Inuit tienen una tendencia a apropiarse de este medio, de hacerlo una herramienta para la promoción de su cultura. Pero cuando todo este dicho y hecho, con lo que nos quedamos son formas de hacer las cosas y retóricas que son esencialmente anglo-europeas en vez de nativas (Ibíd.).

Si bien estos argumentos de Savard y Dicks invitan a reflexionar y complejizan visiones deterministas de la tecnología, el núcleo duro de sus posturas entiende a una cultura como algo estático e inadaptable al cambio. Los argumentos sobre una "metacultura" en Internet son de por si problemáticos ya que investigadores como Neil Blair Christensen $(2003,2006)$ han sugerido que los Inuit efectivamente usan la red para "empotrar" allí su mundo de la vida siendo a su vez una manera para reforzar identidad. De hecho, Christensen (2003: 17) parafrasea a la escritora Inuit Rachel Qitsualik en su idea de que las tradiciones nómades de los Inuit van bien con la movilidad disponible en internet, ya que los "Inuit pueden seguir viajando en un mundo que paradójicamente parecería estar expandiéndose mientras que se encoje”. Este paralelismo describe cómo los Inuit pueden apropiarse de una tecnología como internet de la misma maneras que lo han hecho con muchas otras tecnologías (rifles, como ejemplo más claro) desde los primeros contactos con foráneos. Como sostiene Christensen, Internet 
es usado como "promotor de cultura e identidad en vez de ser un filtro posmoderno que empuja hacia la construcción de nuevas ciberidentidades o ciberculturas" (2003: 18). Esto se relaciona con que hay un fuerte vínculo entre la tecnología y cultura, entre TICs y experiencias locales, como lo expresa Woolgar: "el uso de nuevas tecnologías depende crucialmente del contexto social" (2002: 14), ya que "las tecnologías virtuales suplementan más que sustituyen las actividades reales" (2002: 16).

La tecnología y cultura se interconectan, de modo que aquellas se introducen en un tejido sociocultural preexistente. Como lo subrayan Aporta y Higgs (2005) en su estudio del uso de GPS por cazadores Inuit, las tecnologías son siempre introducidas en contextos sociales más amplios. En este sentido, hoy en día los iglús y trineos de perros coexisten con teléfonos celulares y motos de nieve, donde Internet se ha convertido en una herramienta crucial para mantener el control de las representaciones de los Inuit, y en el caso de Nunavut, para manejar su propio territorio. En este sentido, cultura y tecnología se refuerzan y adaptan mutuamente.

\section{Descifrando la identidad Inuit}

Para entender cabalmente la relación entre el uso de TIC y la identidad Inuit, primero es necesario entender los cambios culturales que los Inuit han sufrido desde su transición de una vida semi-nómada a las comunidades sedentarias de hoy en día. De la misma manera que discutí arriba, la incorporación de diferentes tecnologías ha sido una constante en los cambios más amplios que la cultura Inuit ha sufrido, frente a los cuales han mostrado una particular resiliencia. De este modo, por ejemplo, un Inuk $^{6}$ puede tener una cuenta en Facebook y usarlo para sus propios fines de la misma manera que los rifles fueron incorporados como una herramienta ya necesaria e imprescindible para cazar, o los GPS para orientarse en las expediciones de caza ${ }^{7}$.

Las cosmologías indígenas no diferencian entre naturaleza y cultura, sino que las ven como un todo inescindible (ver, por ejemplo, Ingold, 2000, Basso; 1995; Casey, 1996, Mazzullo e Ingold, 2008). Por un lado, el cambio hacia un modo de vida con ontologías y epistemologías occidentales alteró para siempre la forma de ser Inuit, más si consideramos que el cambio de vivir de forma nómada a su situación actual ocurrió tan solo en una generación. Goehring y Stager (1991) han descrito este proceso como uno extremadamente traumático y de grandes pérdidas. Apuntaban a que los jóvenes Inuit están en riesgo de ser más cercanos a telenovelas o símbolos occidentales que a sus propias tradiciones, y que un anciano "nacido en un iglú, quien aprendió a hacer herramientas de piedra cuando joven, y conoció a su primer Kablunak $^{8}$ de adulto... Ahora vive en un bungalow de estilo suburbano con calefacción central en una comunidad, y pasa parte de su retiro jugando ávidamente al Nintendo y haciendo zapping en la televisión".

De forma similar a las posturas de Dicks y Savard, parecería que Goehring and Stager tienen en mente a Inuit que pertenecerían a un museo, incapaces de cambiar. Estos

Mientras que Inuit es el plural, Inuk se refiere a una persona en singular.

En este sentido, Aporta y Higgs (2005) sostienen en su estudio del uso del GPS que lo cazadores de la comunidad de Igloolik al norte de la isla Baffin hacen, que la tecnología "no está creando un reemplazo de los métodos Inuit y la tecnología per-se no está socavando el ser Inuit de los cazadores de Igloolik” (p. 752).

Kablunak o Qallunaat es el término genérico para referirse a los no Inuit. 
autores otra vez apunan a la tecnología como factor que hacen a los Inuit incapaces de procesar los cambios de manera culturalmente aceptable. El problema de esta postura es que concibe a la cultura como algo estático, reduciéndola así a una entidad esencial. Pero como Christensen (2006: 89) escribe: "A pesar de que la identidad y cultura Inuit suelen ser pensadas en un contexto "museológico" por parte de los extranjeros, sus contenidos y dinámicas siempre han estado sometidos a continuo cambio".

En este marco, la pregunta clave es: ¿Qué significa ser Inuit hoy en día y cuál es el rol de las tecnologías foráneas en una cultura indígena? ¿Son "menos indígenas" cuando tecnologías modernas son introducidas en la vida diaria? Esto refiere a que, como el antropólogo George Wenzel (1991) sostuvo en su estudio sobre la caza de focas, los occidentales hemos definido los parámetros en los cuales analizar la cultura Inuit. Quienes se oponían a la caza de focas argüían que los Inuit "ya son como nosotros", porque "los artefactos que hacían a los Inuit lo que eran ya no son parte visible de su presente" (Wenzel, 1991: 6).

El extenso trabajo del antropólogo Ned Searles (2001, 2002, 2006, 2008, 2010), ayuda a pensar en estas preguntas, ya que se propone identificar los componentes del "ser Inuit". Para ello, se detiene en diversos aspectos como ser el lugar de residencia, la alimentación, o la práctica de actividades tradicionales como la caza. De este modo comenta que los ancianos Inuit creen que el ser Inuit no puede ser desarrollado en comunidades como Iqaluit, ya que allí la identidad está siendo erosionada y carece de autenticidad: "Qallunaat es al establecimiento urbano lo que el Inuit es a la tierra", se dice. Searles afirma que la mejor manera de practicar las costumbres Inuit hoy en día es en los campamentos en las afueras de las comunidades, en donde los Inuit retornan en alguna medida a la vieja forma de ser Inuit en la tierra en contraste a su vida sedentaria dentro de las comunidades.

El trabajo de otros académicos es también orientador en este sentido. Por ejemplo, en sus estudios etnográficos sobre el proceso de modernización de la comunidad de Quaqtak, en Nunavik, al norte de Quebec, Jean-Louis Dorais (1977) ya comentaba que la identidad es un proceso dinámico y creativo donde "un Inuk moderno que es profesor es tan Inuk - si él o ella se percibe como tal - de la misma manera en que un Inuk más tradicional quien pasa sus días cazando focas o cosiendo ropas de piel" (p. 5). En la misma dirección, Jens Dahl (2000) y Pertti Pelto (1973/1987) examinaron las maneras en que las tradiciones de los pueblos indígenas árticos son negociadas con los tiempos que corren. Por ejemplo: la caza o la pesca han sido las prácticas culturales por antonomasia que ahora son negociados con trabajo asalariado durante los días de la semana, o por medio del trabajo en negocios flexibles como co-operativas (ver también McElroy, 2008; Stuckenberger, 2006; Wenzel, 2009).

Esto demuestra como los Inuit están constantemente re-interpretando su identidad para darle sentido a sus vidas, lo cual promueve a la juventud a articular tradiciones en un contexto social constantemente cambiante (Searles, 2001). En esta transformación social los Inuit se movieron de un mundo "impregnado con rituales, relaciones, y sentido, a uno impersonal, secular, y de relaciones mercantiles" (Tester, 2010: 143), donde es importante reconocer que la dinámica cambiante y adaptable de la identidad Inuit y su capacidad de agencia. Graburn (2006: 154) se refiere a este punto cuando reflexiona sobre la identidad Inuit:

Si miramos de cerca al Inuk icónico y real, la imagen aparece ser a la vez un mito y mítica - un modelo rígido, inexacto y pasado de moda, usualmente promovido por 
alguien que clama ser una autoridad.... No obstante, hoy en día hasta la naturaleza del Inuit Qaujimajatuqangit ${ }^{9}$ está siendo desafiada por los Inuit más jóvenes, quienes pueden tener una noción distinta de lo que constituye a un Inuk real. Para ellos, un Inuk real no debe ser juzgado por los estándares de una era más temprana ni ser sostenida como un modelo mítico, desviado, e imposible de obtener hoy en día.

Lo que Graburn hace aquí es enfatizar que la definición de lo que significa ser Inuit cambia. Un Inuit que navega por Internet y usa Facebook, como veremos, para ver lo que sus amigos y familiares están cazando alrededor del territorio no es menos Inuit que otros. Las culturas fluyen y no existe tal cosa como estados culturales puros. Para interpretar correctamente la realidad Inuit, es necesario deshacerse de la ideología occidental de un ártico prístino con gente que vive en iglús y come foca. Como Dorais (1997: 106) escribe, se puede ser Inuit y "moderno" a la vez:

La identidad Inuit incluye la manera específica en que los nativos del ártico establecen sus relaciones con otras personas, animales, la tierra, y todo el universo. Los Inuit sienten una continuidad con sus ancestros, incluso cuando están viviendo en comunidades permanentes, siendo asalariados, yendo a la escuela o a reuniones del gobierno local, o llevando registros en lenguaje Qallunaat. La identidad genuina Inuit se encuentra por lo tanto concomitantemente en lo "tradicional" como en lo "moderno".

De modo similar, Jens Dahl (2000: 39) nota el impacto de las tecnologías de la comunicación en la comunidad groenlandesa de Saqqaq:

Mientras que muchos observadores pueden llegar a tener la impresión de que la tecnología moderna funciona hacia la destrucción de las relaciones e instituciones "tradicionales", yo me atrevería a tomar la posición opuesta en relación a algunos de los medios de comunicación modernos, los cuales agrandan la red espacial como la social y las hacen más eficientes.

En resumen: los Inuit tradicionalmente han incorporado prácticas y tecnologías foráneas adaptándolas para sus fines, y como discuto en este artículo, las TICs no son la excepción. En la próxima sección exploro el uso de la red social Facebook por jóvenes. Facebook emergió en mi trabajo de campo y llamó mi atención en particular ya que los participantes en la investigación la asociaban instantáneamente con las TICs. Al darle los participantes tanta importancia, mi atención se enfocó en el uso de dicha red social. Por ejemplo, uno de los estudiantes me comentó de forma lúdica: "Soy más adicto al Facebook que al alcohol"10. Esta expresión resume bastante bien la intensidad del uso de dicha red social entre los estudiantes en la residencia. Durante mi trabajo de campo observé constantemente como los estudiantes estaban en sus laptops o teléfonos celulares todo el tiempo, durante el desayuno, almuerzo, cenas, en la sala común de televisión, en sus cuartos, o en la cabañita para fumar situada afuera de la residencia. Cuando les preguntaba que hacían con sus aparatos, la respuesta solía ser "estar en Facebook". De hecho, cuando hablábamos sobre tecno-

9 Inuit Qaujimajatuqangit significa la forma tradicional de ser Inuit, o los valores Inuit.

10 Comentario tragicómico, ya que el nivel de alcoholismo en Nunavut es muy elevado. 
logías e internet, la primera palabra que se asociaba era Facebook, como si internet y esta red social fueran sinónimos.

Voy a describir dos categorías de uso: uno individual y otro que refiere a compartir y acceder a contenido identitario-cultural, principalmente por medio de la creación de grupos ${ }^{11}$. El primer uso responde al uso de una tecnología de acuerdo a lo que esta permita a hacer a las personas, o al uso que promueve en un contexto sociocultural determinado. El segundo refiere a un uso particularmente Inuit que demuestra la apropiación de una tecnología para sus propios fines, y lleva a repensar la relación entre aparatos tecnológicos e identidad.

\section{Uso individual de Facebook}

Las expresiones de los estudiantes muestran la relevancia de Facebook en el contexto en el cual es utilizado. Los estudiantes, por ejemplo, hacen referencia a las oportunidades que brinda para estar en contacto con familiares distantes, amigos, y comunidades. En las palabras de un estudiante: "Facebook es útil para saber que está pasando". Otro también decía que "lo uso para estar a tiro con lo que pasa a diario, especialmente eventos en la comunidad, amigos... lo que pasa en la comunidad, hablar con amigos", mientras que otro expresa que lo usa para:

conectarse con familia en todos lados. Para publicar cosas en el muro, normalmente para sorprender a mi familia, contarles donde estas, que es lo que estás haciendo. Un amigo en Rankin Inlet ${ }^{12}$ cazó una beluga ${ }^{13} \ldots$ cosas como esas... sorprendentes. También sigo a un montón de bandas, carreras de moto-cross, 'gamers" árticos. También sigo a "Rankin Inlet elders" y "sell/swap"14. Facebook es una herramienta útil para contarle a la familia cómo estoy, hablar con mi madre quien le dice a mi abuelo.

Facebook fue constantemente mencionado como uno de los principales medios de comunicación en el territorio. Uno de los estudiantes lo subrayó diciendo que usaba internet para: "conectar con familia y amigos en otras comunidades a través de email y Facebook, pero principalmente Facebook". Y otro que apuntaba que su uso de Internet era básicamente para "redes sociales y así estar en contacto con amigos alrededor de Canadá". Facebook proporciona una plataforma para compartir imágenes, por lo que un estudiante decía "Facebook es usado para comunicar si alguien cazó un animal grande, por ejemplo", algo que es usualmente encontrado a través de las sus páginas de Facebook.

Un estudiante mencionó que "subo imágenes porque no quiero perderlas. Me conecto con amigos y familia por el mundo...sigo al grupo de noticias de Pangirtung ${ }^{15}$,

11 Usos que están en línea con lo que Budka (2012) y Budka et al. (2009) demuestran en cuanto al uso de la red indígena MyKnet.org en el norte de la provincia de Ontario.

12 Rankin Inlet es una comunidad en Nunavut.

13 Las belugas son un tipo de ballenas que los Inuit han tradicionalmente cazado y han formado parte de su dieta.

14 Rankin Inlet Elders trata sobre los ancianos de la comunidad mientras que Sell/Swap son sitios usados para la venta y trueque de diferentes cosas. Por lo general cada comunidad de Nunavut tiene el propio.

15 Comunidad en el territorio de Nunavut. 
donde cualquier persona de esa comunidad puede postear lo que quiera, como ser, juntarse para cenar comidas tradicionales. Yo estoy al tanto de lo que pasa en la comunidad cuando veo esa página". Facebook también es usado con otros fines, como lo expresaba una participante que realiza canto de garganta ${ }^{16}$ : "Tenemos una página de Facebook y un canal de YouTube que son una gran manera de comunicarse con la gente. Tu mensaje llega lejos". Y también puede ser utilizado para recibir esos mensajes que hablan de las prácticas tradicionales, como decía una estudiante: "Puedo aprender más de lo que se hacía en los viejos tiempos".

Otro estudiante, por otro lado, se mostró ambivalente con los usos de Facebook. Por un lado menciona que: "Las redes sociales y Facebook tienen un lado bueno y malo. La comunicación social es una de las más grandes cosas que han pasado en Nunavut. Uno enciende su laptop, tu iPod, y podes ver mensajes por todos lados" Pero mostro su lado crítico: "Es también utilizado para buscar drogas, marihuana". Ahí es cuando no me gusta mucho... con gente pidiendo dinero para comprar marihuana." Y agrega que "Antes del 2006 mi vida era mejor. No sabía de Facebook, mis amigos no sabían de Facebook. Nos dedicábamos a jugar afuera al béisbol, ir al gimnasio y practicar deportes, salir con nuestros abuelos. Ahora estoy sentado en esta silla mirando a Facebook. Es una adicción... pero una buena porque también me ayuda."

Facebook es también usado para mantener conversaciones que refieren a la cultura e identidad tradicional de forma individual. Por ejemplo, uno de los participantes escribió en su muro cómo estaba mirando un programa de comedia famoso en la televisión canadiense donde hablaban que los Inuit ya no viven en iglús. De este comentario en su muro recibió otros de otras personas, una de ellas diciendo que le pareció absurdo que en Toronto y Ottawa le preguntaban si los Inuit vivían en iglús. De ahí surgió una conversación en tono burlón de la ignorancia de los otros, y de lo estúpidas que esas preguntas son para ellos.

Asimismo existen usos que corresponden con fenómenos sociales actuales. Uno de los participantes dice: "Yo publico mis propios pensamientos... cada vez que necesito ayuda de mis amigos. Pido un poco de ayuda de mis amigos y familia. Hago a mis amigos sonreír cuando precisan ayuda. Si acusan de algo a un amigo en Facebook, trato de ayudarlo". Esto se relaciona con lo que un Inuk me mostró vinculado a un problema gravísimo que padecen los Inuit: la crisis de suicidios. La persona me mostró en su cuenta como Facebook es utilizado como una forma de recordar a aquellos que se suicidaron. Esto demuestra como las TIC son movilizadas como herramientas de soporte social y resiliencia. En este sentido, Wachowich y Scobie (2010) en su estudio del uso Inuit de YouTube concluyeron que contando historias en ese medio, el contexto de la vida se hace más manejable. Es preciso tener en cuenta que los Inuit padecen la tasa de suicidio más alta del mundo, un fenómeno vinculado al proceso de colonización. Quizás el hacer más manejables las circunstancias adversas de la vida sea el caso con Facebook también, al recordar allí a los que se suicidaron $^{18}$. La red social provee con una herramienta que permite desahogarse y

16 Throat singing en inglés, es una práctica tradicional femenina que consiste en producir sonidos guturales con la garganta que imita a sonidos de la naturaleza. Se suele practicar de a dos.

17 El consumo de marihuana y hachís es frecuente por parte de los jóvenes Inuit.

18 A esto apuntan hallazgos recientes del uso de Facebook por parte de Aborígenes Australianos (ver Carlson et al. 2015), lo cual demanda se investigado para el contexto Inuit también. 
colectivamente recordar. Así es que otro estudiante decía "Facebook me hace feliz e infeliz... cuando veo cosas como que alguien se suicida... lo veo en Facebook. Así que a veces es shockeante... pero Facebook me ayuda a no estar solo."

El uso individual de Facebook parecería indicar que es una tecnología que se suma al tejido social preexistente de los Inuit. Como indico Dorais (2010) en su etnografía en el ártico Quebequense, los Inuit parecerían usar las nuevas TIC como herramientas que prolongan su ser social tradicional, en el sentido de fomentar las redes sociales y su sociabilidad tan característica. Facebook cumpliría esta función en el día a día de los estudiantes participantes. En la próxima sección me detengo en el uso de Facebook para formar grupos.

\section{Uso de grupos de Facebook}

Facebook otorga la posibilidad de crear y mantener grupos los cuales pueden ser abiertos o cerrados. Los abiertos son públicos mientras que a los cerrados se accede por autorización del administrador del grupo. Los estudiantes me demostraron la importancia de estos grupos para su vida diaria y su cultura.

Si uno utiliza el buscador de Facebook y escribe palabras clave como Inuit, Inuk, o Nunavut, puede encontrar una miríada de grupos. Los jóvenes participantes de este estudio constantemente se referían a uno llamada "Inuit Hunting Stories of the Day"19. Este grupo muestra lo que la gente caza y se comparten consejos de caza. Este tipo de grupos fueron reconocidos por los participantes como una buena forma de presentar a la cultura Inuit, de acceder a su propia identidad, de acceder a conocimientos y técnicas tradicionales. Los grupos de Facebook con contenido cultural tienen significados diferentes. Por ejemplo, un estudiante, de manera un tanto defensiva, quizás pensando que yo me oponía a la caza de focas dada la constante oposición a esta práctica por parte de organizaciones Qallunaat (no Inuit) como Greenpeace, dijo: "Nosotros subimos la matanza de animales... eso es lo que hacemos para sobrevivir en el norte. Nosotros no podemos cultivar nada en el ártico". Y agrego que esos grupos son buenos para "mostrarle a la gente cómo cazar correctamente, para enseñarles." De manera similar, otro participante dijo que accedía a "Nunavut Hunting Stories, donde muestran como cazan y que es lo que cazaron", añadiendo que: "mi padre saca fotos de una foca con su teléfono celular, como por ejemplo cuando mi hermanito cazó su primer beluga, y lo subió a Facebook para mostrárselo a todos".

Los grupos fueron también mencionados como importantes para la conservación cultural: "son una buena herramienta porque la gente está perdiendo sus tradiciones. Especialmente si vas a cazar por primera vez puedes mirar la historias de caza y ver qué hacer con distintos animales". Otros, no obstante, recalcaron la relevancia de la comunicación oral: "No lo uso para seguir historias de caza. Prefiero hablar en persona", acto seguido agregando que "Facebook es usado para comunicar si alguien cazo algún animal, por ejemplo. Pero algunas personas no pueden pagar el acceso a internet o a tecnologías, así que la interacción personal es mejor".

La noción de compartir estuvo también presente en términos de compartir conocimientos a través de Facebook: "Yo sigo el grupo de Hunting Stories donde

19 “Historias Inuit de Caza Diarias", sería la traducción del inglés. 
muestran los animales cazados, como osos polares o focas. Puede ser usado para compartir cosas, y mostrarle a mucha gente en internet que es lo que está pasando. Compartir el conocimiento y dárselo a otras personas es bueno, darles conocimiento para que otros lo usen". En palabras de otro estudiante: "Es realmente excelente ver que está pasando y lo que la gente caza alrededor del territorio... en Hunting Stories se comparte mucho conocimiento. Es cultura Inuit para que los Inuit vean y reconozcan que su cultura todavía es fuerte en algunas comunidades. Es interesante que los Inuit podamos compartir nuestro conocimiento sobre caza, guantes Inuit, cuentas de fantasmas Inuit ${ }^{20}$, cosas como esas. Hay un montón de historias sobre nuestra tierra. Incluso tu puedes buscar y terminar tu proyecto por internet! ...Pero tienes que hablar con los ancianos...Internet puede ser usado para acceder toda la historia de los Inuit, como las escuelas residenciales. Las redes sociales también pueden ser utilizadas para compartir esa información. Hunting Stories es el lugar número uno para ir y asociarse con gente para que compartan su conocimiento".

Otro estudiante menciona que su uso de Facebook consiste en: "Nunavut Hunting Stories, mirar las fotos de nuestro territorio, jugar a juegos, estar en contacto con gente... Nunavut Hunting Stories cuando estoy triste... Nunavut Hunting Stories te muestra lugares que te dan ganas de visitar...". También dice que Facebook "es apropiado para la cultura Inuit porque los Inuit dan la bienvenida a gente y les gusta conocer otras personas. Hunting Stories muestra animales y gente y lo que cazaron. Me recuerda a cuando yo iba de cacería, y a veces me enseña como cazar, como sacarle la piel a los animales, y cosas así."

Las redes sociales también pueden ser utilizadas para manejar sus propias representaciones, como propone un estudiante: "[Facebook] me parece una buena cosa porque no mucha gente sabe del norte, y hay estereotipos sobre las comunidades del norte, y no es realmente justo. Más que nada de que somos alcohólicos, que nos drogamos, gueto... la gente que no sabe mucho del norte, eso es lo que escuchan. Si la gente estuviera interesada, podrían saber más del norte." O cuando hable con unas personas que tenían un grupo de Facebook, mencionaron que "No es que estemos representando a los Inuit, pero estamos mostrando como preservamos y en que estamos intentando contribuir. Queremos que la gente aprenda de nosotros, que sean más felices. Es traer los días pasados, felicidad, tradición, y espíritu".

Asimismo hay otros usos para compartir y acceder a la cultura Inuit que los participantes recalcaron, como por ejemplo: "Hay un soundcloud en internet. Escuchamos a muchos artistas usando nuestro lenguaje, cosas muy buenas". Por ejemplo, un video que me mostró un participante fue una versión de una canción de la cantante pop Rhianna en Inuktitut, cantada por una joven Inuk que versiona canciones pop en el lenguaje Inuit tradicional, Inuktitut. Esto es otro indicador de la "colonización" Inuit de internet, ya que la promoción del lenguaje y lograr que las nuevas generaciones practiquen la lengua tradicional es fundamental para la cultura Inuit (asunto discutido por Pasch 2008 y 2010).

Lo que estas citas muestran es el valor de los grupos de Facebook como repositorios de contenido cultural, como una manera de consultar sobre las formas tradicionales de ser Inuit tanto para ellos mismos como para los Qallunaat, así como para manejar sus propias representaciones. Básicamente, los grupos de Facebook

20 Aquí se está refiriendo a grupos en donde se comercializan productos tradicionales como guantes o botas hechos por artesanos Inuit, y a otros grupos Inuit que tratan sobre cuentos sobrenaturales. 
son espacios abiertos y cerrados donde los Inuit pueden juntarse por medio de sus dispositivos para compartir opiniones y pensamientos, donde las referencias a las maneras tradicionales Inuit son usualmente encontradas para diversos propósitos de conservación y comunicación.

Por otro lado, otros sitios populares son las páginas de venta o trueque de diferentes comunidades. Aquí, las personas venden cosas que fabrican como guantes, kamiks $^{21}$, amautis ${ }^{22}$, joyas, o lo que cazan. Se toman fotos del producto o la comida las cuales se muestran en los grupos de Facebook con una breve descripción y el precio base. El artículo es subastado hasta cierta fecha en donde quien ofrece más dinero se queda con él. Varios participantes en este estudio reconocieron que ellos mismos venden sus artesanías o que tienen miembros de su familia que lo hacen a través de estas páginas. Nancy Wachowich (2006) escribió que los Inuit ven a su cultura hecha una "commodity" ya que productos que 50 años atrás eran fundamentales para su supervivencia en la vida diaria ahora son vendidos como artesanías exóticas. Hoy en día, a través de canales como Facebook, los Inuit ofrecen sus productos 24 horas al día para todo el mundo. En el sentido de Wachowich, los Inuit usan las TIC como un canal más para "comodificar" su cultura.

Es útil ver ejemplos de las descripciones de algunos grupos de Facebook que se refieren a asuntos Inuit. Por ejemplo:

- "Mostrar fotos de tus cazas, videos de tus cazas, fotos de tus campamentos, fotos de animales historias de tus cazas, historias de tus encuentros con animales árticos exóticos, historias que tengan que ver con el ártico. ${ }^{23}$

- Recordar o saber sobre cosas que los niños no saben hoy en día. (recordar, saber cosas pasadas).

- Debemos preservar nuestra forma tradicional de vida. Siéntanse libres de agregar a gente y de subir fotos. Por favor, abstenerse de insultos. Que nuestro conocimiento no sea olvidado.

- Este link es para darle a los Inuit conocimientos tradicionales sobre cómo sobrevivir y cómo cazar de manera segura. Espero que la gente pueda contar o compartir historias interesantes. Que nuestra forma de vida sea conocida, y esperemos que esto ayude a otras personas en muchas maneras posibles. Por favor, posteen cualquier consejo de seguridad sobre cómo cazar y sobrevivir en el ártico. Necesitamos el mayor apoyo de nuestros ancianos como sea posible. También quisiéramos pasarle nuestro conocimiento a nuestros niños y jóvenes.

- Si tienes historias sobre cualquier historia tradicional Inuit, cuéntanos lo que sabes, cualquier cosa desde inukpasugjuk, qalupiluks e incluso lo que tus abuelos hacían.

- Como la medicina tradicional, necesitamos empezar a contar historias, dichos, palabras que nuestros ancianos decían porque están siendo perdidas. Hemos perdido demasiado, ahora tenemos una avenida de mantener estos con vida.

\footnotetext{
Botas tradicionales.

Parcas tradicionales.

Aquí es necesario recalcar que este mensaje estaba escrito en inglés y francés (los idiomas oficiales de Canadá) así como en danés, hablado en Groenlandia. Esto hace referencia a una identidad Inuit global, ya que ellos se extienden por Alaska, el norte de Canadá (incluyendo Quebec donde el idioma es el francés), y Groenlandia. Llama la atención, sin embargo, que los idiomas tradicionales Inuit como el Inuktitut o Innuinaktun están ausentes.
} 
- Necesitamos empezar a contar historias, decir las palabras que nuestros ancianos decían porque están siendo perdidas.

- Siempre pensé que esto era una Buena idea que nadie estaba haciendo, nadie que yo conociera al menos, cuentanos tu historia de qalupiluk, como los cuervos tomaron su color negro o inukpasugjuit, o las historias de tus abuelos de cómo sobrevivieron o de lo que sabían".

Estos ejemplos muestran cómo las redes sociales están siendo usadas para vincularse con las tradiciones, consultar a otros por prácticas, fortalecer la identidad, dialogar. Estos extractos apunan a lo que Landzelius $(2006,2003 b)$ ha descrito como usos indígenas "internos" y "externos" de las TIC, que refieren a la vez a un público interno y a otro externo. A través de las TIC, se muestra cómo los Inuit han creado una avenida para comunicar y mantener tradiciones y prácticas a su alcance virtual. Los usuarios ven en estos grupos una oportunidad para examinar que significa ser Inuit, hacer conexiones al pasado al mismo tiempo de compartir e intercambiar ideas con otros. Como Christensen (2003) analizó, los sitios web con contenido Inuit usan internet para colocar identidades offline, online. La meta-cultura que Savard (1998) predecía, no existe. Al contrario: fronteras identitarias parecerían verse formadas. Porque a lo que los grupos apuntan, como se ve en sus descripciones, es para compartir sus experiencias con personas Inuit, mantener su cultura y transmitirla a las nuevas generaciones.

Estos grupos le hablan a dos tipos de personas distintas. Por un lado, a los propios Inuit, y por otro a personas como quien escribe, un Qallunaat, que nunca podría participar en esos grupos en los mismos términos porque no tengo la experiencia de ser Inuit. Aquí el argumento de Christensen se ve reforzado: las fronteras identitarias se reproducen en redes sociales online. Los Inuit participantes son capaces de interactuar allí y discutir esos límites. Esto se relaciona con observaciones de otros investigadores, como por ejemplo el estudio de YouTube de Wachowich y Scobie (2010) donde observaron que funciona como una red social que puede ser utilizada para contar historias a través de las cuales los jóvenes Inuit pueden "eludir las epistemologías y conciencias dominante occidental que tradicionalmente han gobernado representaciones mediáticas de la vida social Inuit....y clamar su propio terreno en el ciberespacio y más allá" (p. 85). El punto aquí es que una red social como Facebook parecería funcionar como un receptáculo de reflexividad de lo que es ser Inuit, en el cual los usuarios conversan y comparten fotos, muchas veces con el objetivo de enseñar a otros, especialmente a los jóvenes, porque como la descripción de uno de los grupos dice: "Necesitamos contar nuestras historias, decir las palabras que nuestros ancianos decían porque las estamos perdiendo".

\section{Conclusión: tecnología e identidad}

Las TIC han sido un componente clave de la globalización (Castells, 1996), y hoy en día son accesibles en todas las comunidades en Nunavut (como ya mostraba el reporte de Infraestructura y Conectividad Aborigen Canadiense de 2004). Como han demostrado muchos estudios sobre su impacto social, la tecnología es un factor que altera le experiencia del ambiente en una relación de mediación: los artefactos son 
adaptados para los propósitos humanos, y consecuentemente las personas cambian sus hábitos en relación a los objetos materiales de su entorno (Verbeek, 2011; Latour, 1992; Ihde, 2009). De este modo es cierto que algunas innovaciones en la vida de los habitantes del ártico han provocado cambios culturales no del todo auspiciosos que van en detrimento de saberes y costumbres milenarias. Por ejemplo, Aporta (2009) describe como el uso de motos de nieve afectó la comunicación oral mientras se trasladaban en contraste al uso de trineos de perros. La razón es que las motos de nieve hacen ruido que impide que un padre o anciano le hable a un joven enseñándole sobre técnicas tradicionales de orientación que hacen la diferencia entre la vida y la muerte en el frío ártico, o cuando se altera la percepción del ambiente debido a que los perros perciben situaciones de peligro como condiciones del hielo cambiantes (Ingold y Kurtilla, 2000). Similarmente, el uso del GPS ha traído problemas cuando los Inuit pierden la habilidad de orientarse cuando se congela o las baterías fallan en el medio de la tundra, y las habilidades de orientación tradicionales han sido perdidas (Aporta y Higgs, 2005).

Como hallé en mi investigación, las TIC han tenido un gran impacto con los jóvenes Inuit. Han cambiado las maneras de comunicarse, las interacciones, y sus relaciones con su identidad y ambiente. Mencioné arriba las visiones de aquellos que pensaron en el impacto de las TIC en el ártico 30 o 40 años atrás, como ser Savard y Dicks, quienes promulgaban una suerte de distopía, arguyendo que estas herramientas tendrían efectos negativos en la cultura Inuit. Apuntaban a problemas de "occidentalización" y de desorientación cultural. Más recientemente, Krone (2008, 2007) apuntó que las TIC poseen intrínsecamente un "guion" (Akrich, 1992) occidental dado que el software incorporado a estas tecnologías conlleva expectativas occidentales de relacionarse con el mundo. Otros académicos (como ser Dyson y Underwood 2006; Gearherad 2005; Landzelius, 2006, 2003a), sin embargo, han indicado que la arquitectura hiper-textual de internet podría ser interpretada como compatible con la manera en que las poblaciones indígenas se relacionan con el mundo. Las redes sociales, por ejemplo, traen una nueva manera de interacción y actividades que se pueden llevar a cabo en la interfaz de Facebook, como compartir imágenes y chatear. Otros investigadores como Louis-Jacques Dorais (2010: 63) en su estudio sobre la identidad en la comunidad Inuit de Quaqtaq en la porción ártica de la provincia de Quebec, observó el uso frecuente de la red social Bebo (anterior a Facebook) y MSN y escribió: "lo que parece ser "moderno", puede ser, en muchos casos, una nueva manifestación de formas tradicionales de comportarse. Los Inuit han sido siempre muy sociables, por lo que chatear en Internet - que más que nada envuelve a navegadores Inuit - puede ser considerado una extensión de la manera tradicional de hacer redes sociales". Asimismo es importante recordar la importancia que internet tiene en vastos territorios como Nunavut. Como lo subrayaron los participantes en la investigación, es común que la gente se mueva de una comunidad a otra dentro del territorio, por lo que un sitio como Facebook es útil para chatear, lo cual es un uso muy importante por los estudiantes Inuit de la residencia del Arctic College.

Para terminar, quiero subrayar la importancia que tiene hoy en día la tecnología para ser Inuit. De hecho, como discutí más arriba, sería iluso y equivocado concebir algo como una identidad Inuit estática y pura. Las tecnologías son incluidas como parte de la identidad en un proceso fluido y dinámico. La vida social es el resultado del entrelazado inseparable entre humanos y tecnología (Latour, 1993). Entonces, la 
naturaleza de lo social en general podría estar representada como una red compuesta de agentes humanos y no-humanos, formando lo que John Law (1987) denominó "redes heterogéneas". En ellas, la tecnología funciona como mediadora entre los humanos y la realidad (Verbeek, 2011). Al entender la tecnología de esta manera podemos ver la relación entre la cultura Inuit y las TIC de una forma reveladora. Facebook, en este análisis, es un actor en la red heterogénea de la identidad Inuit. Es un factor que da forma a como la cultura Inuit es practicada y experimentada por los Inuit (y los Qallunaat). El ser Inuit incluye hoy en día el compartir contenidos en los grupos de Facebook lo mismo que cazar focas y construir iglús. En otras palabras, lo que significa ser Inuit incluye a internet como un agente de conservación y transmisión de las prácticas tradicionales. La identidad Inuit estaría formada por una red heterogénea que incluye satélites, laptops, teléfonos celulares, motos de nieve, rifles, balas, focas, belugas, etcétera. Cuando un cazador Inuit toma su teléfono o cámara y lo lleva para sacar fotos de la caza para subir a Facebook, su identidad se refuerza. Así es como Facebook aparece ser un actor importante en como los Inuit practican y comparten su identidad. El punto final es que la identidad Inuit tiene que ser concebida incluyendo a las TIC en general, y Facebook en particular, como piezas fundamentales.

\section{Bibliografía}

2004 Report on Aboriginal Community Connectivity Infrastructure, disponible en http:// www.aboriginalcanada.gc.ca/connectivity. May 2, 2005.

Akrich, M. (1992). The de-scription of technical objects, en W. Bijker \& J. Law (eds.), Shaping technology/building society: Studies in Socotechnical Change, Cambridge, MA: MIT Press, pp. 205-224.

Amagoalik, J. (2014). "The New Addiction", Nunatsiaq News, disponible en: http://www. nunatsiaqonline.ca/stories/article/65674my_little_corner_of_canada_nov._28/

Alexander, C.; Adamson, A.; Daborn, G.; Houston, J.; Tootoo, V. (2009) Inuit Cyberspace: The Struggle for Access for Inuit Qaujimajatuqangit. Journal of Canadian Studies, 43 (2), pp. $220-245$.

Alexander, C. (2011) From Igloos to iPods: Inuit Qaujimajatuqangit and the Internet in Canada, in Patricia Randolph Leigh (ed.) International Exploration of Technology Equity and the Digital Divide: Critical, Historical and Social Perspectives, IGI Global, pp. 80-105.

Aporta, C. (2009). The Trail as Home: Inuit and Their Pan-Arctic Network of Routes, Human Ecology, Volume 37: 131-146.

Aporta, C. y Higgs, E. (2005) Satellite culture: global positioning systems, Inuit wayfinding, and the need for a new account of technology. Current Anthropology 46 (5); pp. 729-753.

Basso, K. H. (1996) Wisdom sits in places: Notes on a western Apache Landscape, en Feld, S. and K.H. Basso Senses of place, School of American Research, Santa Fe, pp. 53-90.

Budka, P., Bell, L. B., Fiser, A. (2012) "We Were on the Outside Looking In" MyKnet.org - A First Nations Online Social Environment in Northern Ontario, en Andrew Clement, Michael Gurstein, Graham Longford, Marita Moll, Leslie Shade (eds.) Connecting Canadians: Investigations in Community Informatics, AU Press, Athabasca University: Edmonton.

Carlson, B. L., Farrelly, T., Frazer, R., y Borthwick, F. (2015). Mediating tragedy: Facebook, aboriginal peoples and suicide. Australasian Journal of Information Systems, 19. 
Carpenter, P, Gibson, K, Kakekaspan, C, O’Donnell, S. (2013). How Women in Remote and Rural First Nation Communities are Using Information and Communication Technologies (ICT). Journal of Rural and Community Development8, 2: 79-97.

Casey, E. S. (1996). How to get from space to place in a fairly short stretch of time, in Feld, S. and K.H. Basso (eds.) "Senses of place," School of American Research, Santa Fe, pp. 13-52.

Castells, M. (1996). The rise of the network society. Vol. 1 of The information age: economy, society and culture. Massachusetts and Oxford: Blackwell.

Charmaz, K. (2006). Constructing Grounded Theory. A Practical Guide Through Qualitative Analysis. SAGE Publications.

Christensen N. B. (2006). A screen of snow and recognition reigned supreme? Journeys into the homeland of a Greenlandic webpage, en Kyra Landzelius (ed.) Native on the Net: Indigenous and Diasporic Peoples in the Virtual Age, Routledge: New York, pp. 80-96.

Christensen, N. B. (2003). Inuit in Cyberspace, Copenhagen, University of Copenhagen, Museum, Tusculanum Press.

Damas, D. (2002). Arctic Villagers: The transformation of Inuit settlement in the Central Arctic. McGill-Queen's University Press: Montreal.

Dicks, D. (1977). From Dog Sled to Dial Phone: A Cultural Gap? Journal of Communications, 27(4): 120-29.

Dorais, L. J. (1997). Quaqtaq: Modernity and Identity in an Inuit Community. University of Toronto Press: Toronto.

Dorais, L. J. (2010). Taitsumaninitaanit Siarumut: Language, Community, Identity, and the Young Inuit in Quaqtaq. Centre Interuniversitaire d'etudes et de recherches autochtones (CIÉRA), Faculte de Sciences Sociales, Université Laval.

Dyson, L. E., y Underwood, J. (2006) Indigenous People on the Web. JTAER, 1(1), 65-76.

Dyson, L. E., Hendriks, M. A., \& Grant, S. (2007). Information technology and indigenous people. IGI Global.

Dyson, L. E., Grant, S., y Hendriks, M. (2015). Indigenous People and Mobile

Technologies (Vol. 31). Routledge.

Ess, C. (2005) Can the local reshape the global? Ethical imperatives for humane intercultural communication online, en J. Fruhbauer, R. Capurro, and T. Hausmanninger (Eds.). Localizing the Internet: Ethical aspects in an intercultural perspective. Schriftenreihe des ICIE, Bd 4. Munich, Germany: International Center for Information Ethics.

Fair, R. (2000). Becoming the white man's Indian: An examination of Native American tribal web sites. Plains Anthropologist, 45(172), 203-213.

Gearheard, S. (2005) Using interactive multimedia to document and communicate Inuit knowledge. Etudes/Inuit/Studies, 29(1-2): 91-114.

Geertz, C. (1998). Deep hanging out. New York Review of Books 45 (16): 69-72.

Gibson, James J. (1977). The theory of affordances. En Perceiving, Acting, and Knowing: Toward an Ecological Psychology, ed. Robert Shaw and John Bransford, 67-82. Hillsdale, NJ: Lawrence Erlbaum.

Ginsburg, F. (1991) Indigenous Media: Faustian Contract or Global Village? Cultural Anthropology, 6(1), 92-112.

Gohering, B, Stager, J. (1991) The Intrusion of Industrial Time and Space into the Inuit Lifeworld: Changing Perceptions and Behavior. Environment and Behavior, Vol. 23, No. 6: 666-679.

Graburn, N. (2006) Culture as Narrative, en Pamela Stern and Lisa Stevenson (eds.) Critical Inuit Studies: An anthology of contemporary Arctic Ethnography, pp. 139-155. University of Nebraska Press: Lincoln and London. 
Ihde, D. (2009). Postphenomenology and technoscience: The Peking University lectures. SUNY Press.

Ingold, T. (2000). The perceptions of the environment: Essays in livelihood, dwelling and skill. Routledge, London.

Ingold, T. y Kurtilla, T. (2000). Perceiving the environment in Finnish Lapland. Body and Society, 6 (3-4): 183-196.

Krebs, A. (2011). 'Number One Tool' for First Nations? Facebook. The Tyee (May 30, 2011), disponible en: http://thetyee.ca/Mediacheck/2011/05/30/FNFacebook/print.html

Krone, O. (2008) The Arctic and challenges of the exterior-Knowledge, ICT, and Autonomy. Position paper for the $5^{\text {th }}$ NRF open Assembly, September $24^{\text {th }}-27^{\text {th }}$ 2008, Anchorage, Alaska.

(2007). ICT in the Arctic - Two Elements for Consideration, en Knowledge and Power in the Arctic-Conference proceedings, Rovaniemi 16-18 April 2007.

Landzelius, K. (2006). Native on the Net: Indigenous and Diasporic Peoples in the Virtual Age. London/NewYork: Routledge

(2006) Introduction: Native on the Net, en Kyra Landzelius (ed.) Native on the Net: Indigenous and Diasporic Peoples in the Virtual Age. London: Routledge, pp. 1-42.

(2003a) Mapping the Unfathomable Frontiers of Indigenous Cyberspace: A Survey of the Expanding/Contracting Boundaries of Going Native on the Net.Uppsala University 2003. Disponible en http://publications.uu.se/journals/1402-229X/netverket.htm\#11.

(2003b) Paths of Indigenous Cyber-Activism. Indigenous Affairs, 2: 6-13.

Latour, B. (1993) We Have Never Been Modern, Cambridge, MA: Harvard University Press. (1992). Where are the missing masses? The sociology of a few mundane artifacts, en W.E. Bijker and J. Law (eds.) Shaping Technology/Building Society: Studies in Sociotechnical Change, edited by. Cambridge, Mass.: MIT Press.

Law, J. (1987) Technology and heterogeneous engineering: the case of Portuguese expansion, en Wiebe E. Bijker, Thomas P. Hughes and Trevor J. Pinch (eds.), The social construction of technological systems: new directions in the sociology and history of technology, Cambridge, MA: MIT Press, pp. 111-34.

Mazzullo, N. e Ingold, T. (2008). Being Along: Place, time and movement among Sami People, en Baerendholdt, J. and Granas (eds.) Mobility and Place: Enacting Northern European Peripheries, Ashgate, Hampshire, pp. 27-38.

McElroy, A. (2008). Nunavut Generations: Change \& Continuity in Canadian Inuit Communities. Waveland Press, Inc.: Long Grove, Illinois.

McMahon, R. (2013) "Digital Self-Determination: Aboriginal Peoples and the Network Society in Canada." Doctoral dissertation, Simon Fraser University (2013). Disponible en

http://summit.sfu.ca/system/files/iritems1/13532/etd7913_RMcMahon.pdf.

Pasch, T. (2010). Starting Fire with Gunpowder revisited: Inuktitut New Media content creation in the Canadian Arctic. Etudes/Inuit/Studies, 34(2): 61-80.

(2008). Inuktitut Online in Nunavik: Mixed-Methods Web-Based Strategies for Preserving Aboriginal and Minority Languages. Doctoral Thesis, University of Washington.

Pelto (1983) The Snowmobile Revolution: technology and social change in the Arctic. Waveland Press: Prospect Heights, Illinois.

Rodgers, K. y Scobie, W. (2014) Contestations of resource extraction projects via digital media in two Nunavut communities. Etudes Inuit/Inuit Studies, 37(2), 83-101.

Rogers, S., DeGagne, M., Dewar, J. y Lowry, G. (2012). Speaking my truth: Reflections on reconciliation and residential schools. Ottawa: Aboriginal Healing Foundation.

Santo, A. (2004) Nunavut. Inuit television and cultural citizenship. International Journal of Cultural Studies, 7 (4), pp. 379-397. 
(2008) Act Locally, sell globally: Inuit media and the global cultural economy. Continuum: Journal of Media and Cultural Studies, 22 (3), pp. 327 - 340.

Savard, J. (1998) A Theoretical Debate on the Social and Political Implications of Internet Implementation for the Inuit of Nunavut. WicazoSa Review, Vol. 13, No. 2, Technology and Native American Culture (Autumn, 1998), pp. 83-97.

Scobie, W. y Wachowich, N. (2010) Uploading Selves: Inuit digital storytelling on YouTube. Études/Inuit/Studies, 34 (2), 81-105.

Searles, E. (2010) Placing Identity: Town, Land, and Authenticity in Nunavut, Canada. Acta Borealia, Vol. 27, No. 2: 151-166.

(2008) Inuit Identity in the Canadian Arctic. Ethnology, Vol. 47, No. 4: 239-255.

(2006). Anthropology in an era of Inuit empowerment, en P. Stern \& L. Stevenson (eds.), Critical Inuit Studies: An Anthology of Contemporary Arctic Ethnography, Lincoln, NB: University of Nebraska Press, pp. 89-104.

(2002) Food and the making of Modern Inuit Identities. Food \& Foodways, 10: 55-78.

(2001) Fashioning Selves and Tradition: Case Studies on Personhood and Experience in Nunavut. The American Review of Canadian Studies. Spring/Summer 2001: 121-136.

Soukup, K. (2006) Report: Travelling Through Layers: Inuit Artists Appropriate New Technologies. Canadian Journal of Communication, Vol. 31: 239-246.

Srinivasan, R. (2006) Indigenous, ethnic and cultural articulations of new media. International journal of cultural studies, Volume 9(4): 497-518.

(2012) Rethinking Digital Cultures and Divides: The Case for Reflective Media. The Information Society, 28: 24-36.

Stuckenberger, A.N. (2006) Sociality, temporality and locality in a contemporary Inuit community. Etudes/Inuit/Studies, 30(2): 95-111.

Taylor, A. (2011). Social Media as a Tool for Inclusion. Resources and Skills Development Canada. Stiles Associates Inc.

Taylor, A. (2012) Information and Communication Technologies and New Indigenous Mobilities? Insights From Remote Northern Territory Communities. Journal of Rural and Community Development, 7, 1: 59-73.

Tester, F. y McNicoll, P. (2004). Isumagijaksaq: mindful of the state: social constructions of Inuit suicide. Social Science \& Medicine, 58: 2625-2636.

Tester, F. (2010). Mad dogs and (mostly) Englishmen: Colonial relations, commodities, and the fate of Inuit sled dogs. Etudes/Inuit/Studies, 34(2): 129-147.

Underwood, J. y Dyson, L. (2006). Indigenous People on the Web. Journal of Theoretical and Applied Electronic Commerce Research, Vol. 1, Issue 1: 65-76.

Verbeek, P. P. (2011). Moralizing technology: Understanding and designing the morality of things. University of Chicago Press.

Wachowich, N. (2006) Cultural survival and the trade in Iglulingmiut traditions, en Stern, P. and Stevenson (eds.) Critical Inuit Studies: An Anthology of contemporary Arctic ethnography, University of Nebraska Press, Lincoln, pp. 119-138.

Wenzel, G. W. (1991). Animal rights, human rights: Ecology, economy and ideology in the Canadian Arctic. University of Toronto Press.

(2009) Canadian Inuit subsistence and ecological instability - if the climate changes, must the Inuit? Polar Research, 28: 89-99.

Wilson, R, Gosling, S. y Graham, L. (2012). A Review of Facebook Research in the Social Sciences. Perspectives on Psychological Science, 7(3): 203-220.

Woolgar, S. (2002) Introduction: Five Rules of Virtuality, en Steven Woolgar (ed.): Virtual Society? Technology, Cyberbole, Reality. Oxford University Press: Oxford. 
Young-Ing, G. (2003) Perspectives on the Indigenous tradition/New technology interface. Indigenous Affairs 2: 14-17. 